\title{
Implication of Utilizing Phytoestrogens Infested Fodder on Fertility and Histological Structure of Ovaries and Oviduct in Sow
}

\author{
Liviu Marian BOGDAN ${ }^{1}$, Sidonia BOGDAN ${ }^{1}$, Flavia RUXANDA ${ }^{1 *}$, Vasile RUS ${ }^{1}$, Bianca MATOSZ ${ }^{1}$, Viorel \\ MICLĂUȘ ${ }^{1}$ \\ ${ }^{1}$ Faculty of Veterinary Medicine, University of Agricultural Sciences and Veterinary Medicine Cluj- \\ Napoca, Romania. \\ *corresponding author: flavia.ruxanda@gmail.com
}

Bulletin UASVM Veterinary Medicine 74(1) / 2017,

Print ISSN 1843-5270; Electronic ISSN 1843-5378

DOI:10.15835/buasvmcn-vm: 12470

\begin{abstract}
Reproductive efficiency is an essential requirement for the profitability of swine farms and the quality of fodder can have a negative impact on this aspect. We carried out investigations regarding the influence of fodder quality on reproductive efficiency and the histological structure of the ovary and oviduct in 45 sows from a farm in Bihor county. We observed that corn utilized in feeding the sows was stored in inadequate conditions which allowed the development of moulds. The combined fodder, resulted after grinding the cereals, was stored directly on the concrete floor in inappropriate areas, concerning the hygiene. The animals were given green fodder directly on the stall's floor, which favours mould expansion. Upon histological examination, the ovary presented an exaggerated activity and there was a marked congestion in the oviduct, with a tendency of the epithelium toward pseudostratification. The precarious conditions of cereal and combined fodder storing and administration of green fodder, brought optimum conditions for mould development. Under the action of the latter factors, the reproductive performance drastically decreased along with compromising the sows used for reproduction.
\end{abstract}

Keywords: fodder, mould, reproduction, sow.

\section{INTRODUCTION}

For swine breeding, the reproductive activity is decisive in ensuring an adequate reproductive activity, being sometimes influenced at a large extent by certain substances that mimic estrogen hormones. Phytoestrogens are such substances with hormonal action encountered in some fodders, sometimes in large quantities. There are various studies highlighting the negative action of phytoestrogens on reproductive system in some animal species. Nonetheless, some authors sustain that the infertility is temporary and the condition is reversible if the consumption of such fodder does not extend on a long period of time, everything getting back to normal in a month after ceasing the administration of estrogenic-effect fodder (Adams, 1995).

Other substances with hormonal action are produced by various moulds. Moulds are parasitic plants with ubiquitous presence in animal food. Besides the fact that they reduce fodder quality, they synthesize chemical substances termed mycotoxins, which cause great economic losses to animal breeders, especially by drastically disturbing the reproduction activity. Zearalenone is a mycotoxin produced by various moulds from Fusarium genus. Their substrates are: corn, wheat, barley, oat and sorghum. Chemically, it is a non-steroidal compound with a similar action to estrogens from various farm animal species, among 
which we mention swines, bovines, ovines etc. (Hussein and Brasel, 2001). The phytoestrogenic activity is due to the presence of a phenolic nucleus in its chemical structure, as well as its affinity for some of the estrogenic receptors (ERa and ERb), present in some hormonodependent organs in mammals (Shier et al., 2001). Zearalenone can also have an effect on hypothalamic-pituitary axis (Zinedine et al., 2007).

The majority of the researchers claim the fact that the sensibility to zearalenone is different depending on the species, even if their opinion does not always coincide in every aspect. Opinions are unanimous regarding the swine sensitivity. Among them, the prepuber ones are obviously more sensitive than the adults. More frequently, zearalenone determines disorders with clinical signs in prepuber females, but sometimes it induces prepuce enlargement after neutering. Physiological state is also a decisive factor, cyclic females being more sensitive than pregnant sows. Upon natural exposure (in field conditions) to Fusarium moulds, abortion cases were noticed, but experimental attempts with purified zearalenone remained insuccessful (Mirocha and Christensen, 1974). However, scientists observed that zearalenone in concentration higher than 50$100 \mu \mathrm{g} / \mathrm{kg}$ in swine alimentation has a negative effect on the cycle, ovulation and implantation. It can cross the placental barrier causing embryonic death, inhibition of fetal development and a small number of fetuses. Even farrowed piglets have low weight and viability. Zearalenone particularly exercises its harmful actions on the early stages of embryonic development, which is why the first 7-10 days post fertilization are the most critical period of gestation, from this point of view (Diekman and Green, 1992).

The purpose of this study was to highlight the influence of fodder quality and storage on the morphology of ovary and oviduct on sows.

\section{MATERIAL AND METHODS}

The study was carried out in a farm in Bihor county, with a total headcount of 137 pigs, among which 45 sows. The farmers were facing reproduction problems, repeated oestrus, poorly expressed oestrus, small number of farrowed piglets (average of 3 pigs/sow), decreased fertility ratio $(48.8 \%)$. The growth and development performance of the piglets was very poor. The offsprings had hiperkeratosis and the gilts presented tumefaction of vulva. Part of the farrowed piglets remained hipotrepsic, inducing great economical loss.

We harvested samples from the genital system through ovariectomy (the classical method) from sows that were not pregnant after 3 artificial inseminations. The ovary and oviduct were processed in order to perform the histopathological examination. The samples were fixed in $10 \%$ buffered formalin immediately after being harvested and subsequently dehyrated in increasing concentrations of alcohol. We clarified the samples in n-butanol, then embedded them in paraffin in order to section them at $5 \mu \mathrm{m}$ thickness. We performed Goldner's trichrome staining procedure and examined the slides with an Olympus BX 41 light microscope, capturing images with an Olympus E330 digital camera and processing them with Adobe Photoshop CS2 software, version 9.0.1.

We also verified the source and administration of the fodder. The stock areas for the fodder were assessed regarding their capacities and especially the storing conditions. We granted special attention to the way of producing, storing and administration of the combined fodder, obtained by the owner.

\section{RESULTS AND DISCUSSION}

The farm maintenance conditions were far from the necessary standards of the species and physiological situation, for a farm based on reproduction activities. From harvesting to utilization, cereals were stored inside the farm. Corn was stored and kept as cobs in granaries situated in open air, made out of wooden or metalic profiles with wire net, on wood foundation situated directly on the ground, and the shed roof was built from either polycarbonate panels or old tinplates which seemed recycled from other roofs. Because of the storing condition, corn appeared modified, especially in the inferior part of the barn, where corncobs were not yellow anymore, but had a whitish, grayish-white or even grey tint (Fig. 1).

The owner prepared the mixed fodder by himself and had an own recipe mainly based on corn and barley. After milling, the fodder was kept on the concrete floor until it was used to feed the animals. In the area assigned to produce the mixed fodder, the hygiene conditions were quite 


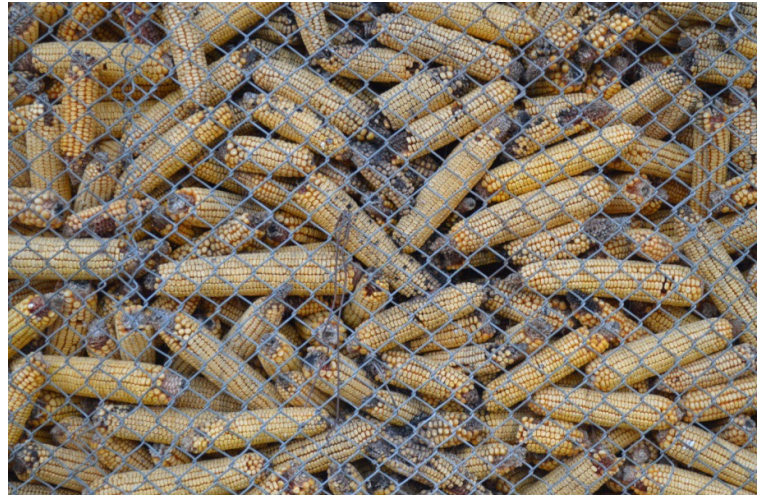

Fig. 1. Corn with moulds

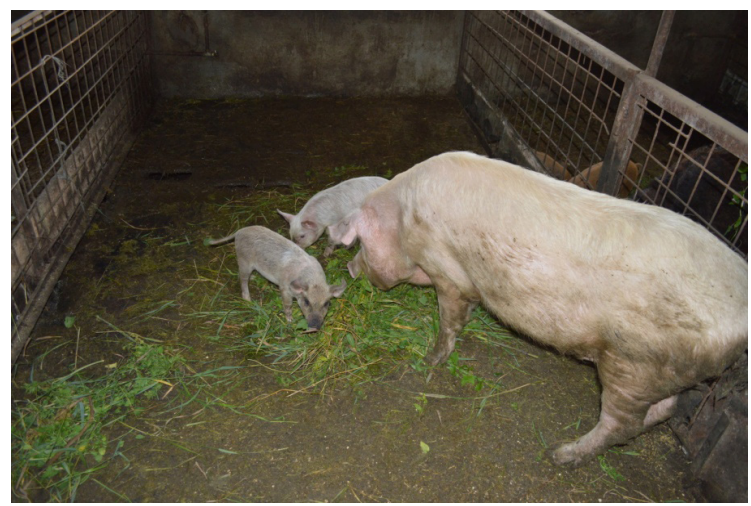

Fig. 3. Sow with 2 piglets

doubtful, the obtained fodder after milling was collected directly on the concrete floor, which presented obvious moisture traces, which seemed to derive from pluvial waters infiltrated through the roof (Fig. 2).

Adult animals were pretty uneven regarding both their size and aspect, so we could estimate that the head count was relatively polymorph. Thus, there were sows that farrowed two piglets (Fig. 3), others with three, while others had 4-5 piglets and rarely more.

In some cases, besides the fact that a small number of piglets per farrow was obtained, there was also an unjustified irregularity between the sows that farrowed a small number of pigs. Moreover, there were cases in which beside the fact that a small number of pigs was obtained, most of them died in first 10 days after birth (Fig. 4).

The histological examination revealed an exaggerated ovarian activity, with an ovarian cortex filled with follicles, distributed throughout the

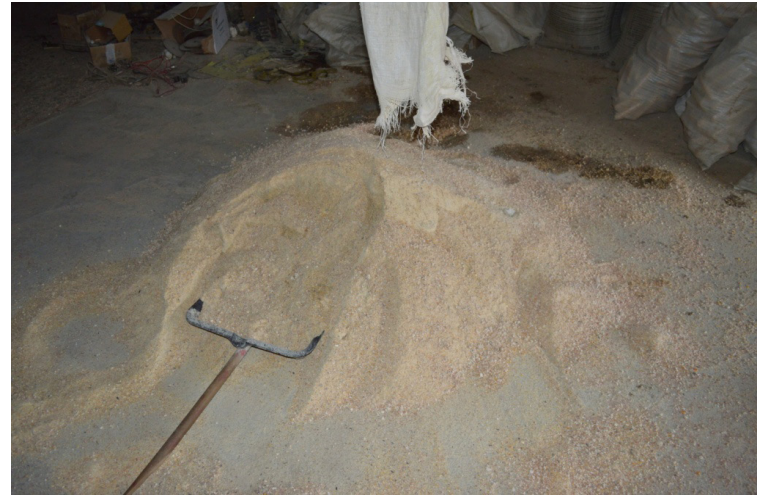

Fig. 2. Water infiltrations in the combined fodder collecting area

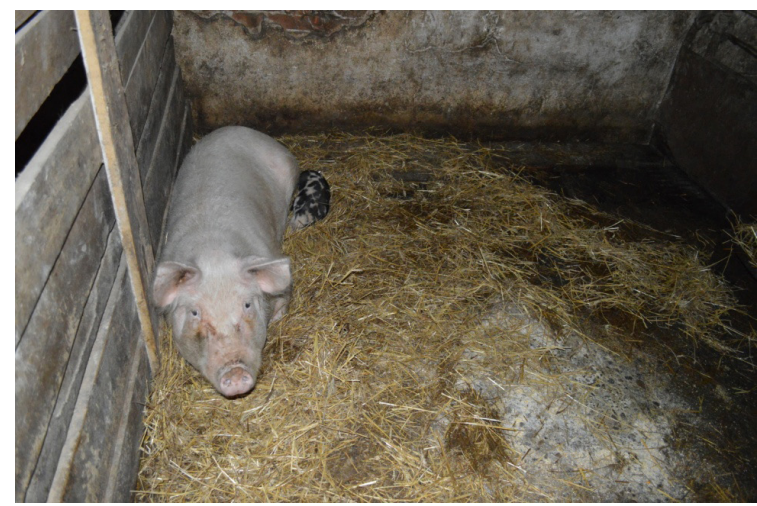

Fig. 4. Sow that farrowed 3 piglets and has only one left

whole section surface of the ovary. Follicles were in different developing and especially involuting stages, but without an ovulation tendency (Fig. 5). There were some follicular cysts present on the surface section, in which the oocyte degenerated. There was no developing or involuting yellow body highlighted, which demonstrates that the situation lingered for a certain period of time. The only plausible explanation is an exaggerated and persistent estrogenic stimulation caused by the mycoestrogens elaborated by the moulds in the fodder.

The oviduct presented a marked congestion and a pseudostratification tendency of the epithelium on relatively large areas without a generalized aspect. We also observed the increase of the ciliated cells' numbers, which appeared in obviously larger numbers than the non-ciliated ones (Fig. 6).

Regarding the assessment of the fodder utilized in pigs' alimentation, there were certain aspects that came to light. According to the shape 


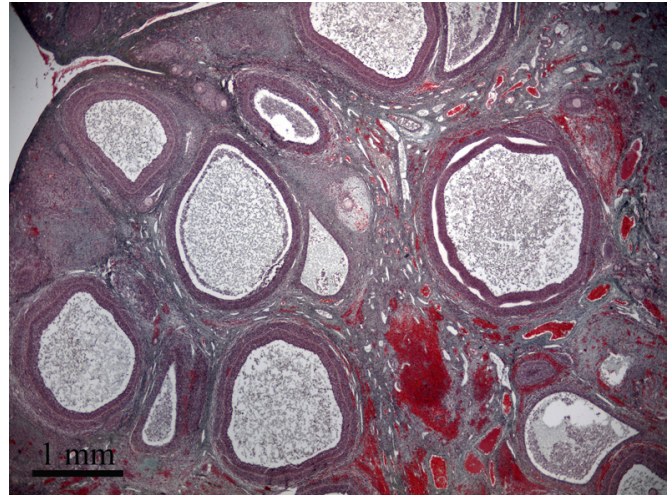

Fig. 5. Exaggerated ovarian activity and generalized congestion ( $2 \mathrm{x}$ obj. - Goldner's trichrom stain)

and size of the corncobs, we can conclude that at the time they were brought at the farm, they had a good quality. The storing conditions in the farm are far from optimal in order to ensure the maintainance of nutritive qualities and keep it safe from bacteria and moulds. The granaries in which the corn is stored are improvised and do not provide optimal storing conditions from various points of view. Firstly, the shed roof with very short eaves only assures partial protection against the humidity from rain, thus the corn on the outside area will periodically come in contact with rain water. The very low foundation, made out of wood, situated directly on the ground, does not provide necessary ventilation for drying and maintaining the corn dry. Humidity easily passes through direct contact, from the ground to the wooden foundation, then to the baseboards forming the floor of the granary and eventually to the corn sitting on them. Furthermore, the ground on which these granaries are placed is humid both because of the rains and the lack of ventilation caused by the direct disposition of the granary on the ground. The presence of humidity is also revealed by the plants growing in close vicinity of the granary and some of them get in direct contact with the materials from the granary's wall or even the corn. Additionally, in granaries with wooden walls, we could observe that some boards are covered in moss on a relatively large area, which actually grows at the expense of humidity in the wooden material. The excessive humidity and lack of ventilation in the lower part of the granary predispose to development of moulds in corn, starting from the base and continuing up to the middle of the granary height or even upper.

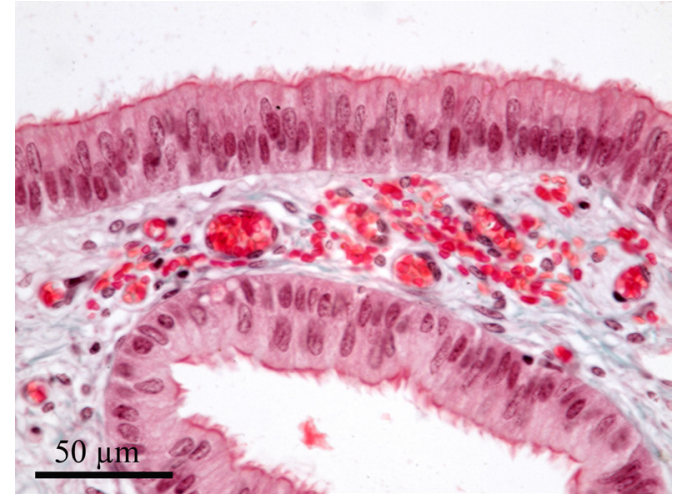

Fig. 6. Pseudostratification tendency of the epithelium and a large number of ciliated cells (60x obj. - Goldner's trichrom stain)

In specialty literature, there are data concerning the risk of contamination of fodder with moulds. Thus, Desjardins (2006) affirms that the fungi genuses that produce mycotoxins with importance in sow reproduction are Aspergillus spp., Fusarium spp. and Claviceps spp. Furthermore, he states that the fungi themselves are not pathogenic for pig, but the mycotoxins they produce are. Due to the negative effects on the reproductive efficiency, the author mentioned above terms the conditions accompanied by the decrease of reproductive performances: reproductive mycotoxicoses. Also, Kanora and Maes (2009), affirm that the development of moulds can take place in different moments (stages) of the plants production process and animal breeding. Mycotoxins can even invade the seeds on the field (before harvesting) or the development of the mould can take place while storing at the farm or combined fodder factory (Desjardins, 2006; Kanora and Maes, 2009). Equally, moulds can develop during fodder processing, in insufficiently cleaned storing areas, means of transport and distribution of fodder, in improvised and inappropriately maintained accommodating areas, and even in the feeding area. Development of moulds and mycotoxin production is amplified by favouring factors such as substrate humidity (10 to $20 \%$ ), relative humidity ( $>70 \%$ ), temperature $\left(0\right.$ to $50^{\circ} \mathrm{C}$, depending on the fungus species) and oxygen availability (Desjardins, 2006; Kanora and Maes, 2009).

Regarding the information in the specialty literature we can state that the risk of fodder contamination with moulds is very high. Avoiding the presence of mycotoxins in food ingredients 
can be difficult; thus, the prevention seems to be highly important. It is crucial to take measures in order to reduce to minimum the possible risk of fodder contamination with moulds. Once they get into animal food, they can determine different effects that can not be controlled or corrected, only to a small extent.

Analyzing the situation in the farm taken into study, we consider that there are many work points in which the moulds find favouring conditions to develop. They involve the width of the granary, which is usually best to be no more than 80 $\mathrm{cm}$, while here it is almost double; under these circumstances, the corn in the middle area is not exposed to sufficient air. Then, the placement of the granary on wooden planks, in direct contact to the ground, determines the maintainance of a high humidity, which highly favours the development of moulds. The area where the combined fodder is produced is maintained in precarious conditions and has water infiltrations through the roof, which maintains a humid state on the floor, where the fodder is kept after milling. Furthermore, inside the shelter, we can observe that neither the walls, nor the floor are maintained so that they do not favour mould development. The feeding area does not look very good and does not seem to be cleaned daily. We can state that in this farm, there are unfortunately favourable conditions for moulds in all work points and the farmer is not aware of the risks he is exposing to and the prejudices that can arise because of this matter.

The ceiling shows that not only was it not bleached in a long time, but the irregular and peeled, stained aspects suggest the fact that rain water infiltrates at this level. This aspect highlights the fact that the roof has various problems which although are not that significant, favour the water infiltration, where they maintain a humid state for a long period of time, which in turn favours mould development, especially during warm seasons of the year. Even though animals do not come in direct contact with the roof, moulds present here can extend through continuity to walls or fall as spores in accommodation areas. In some areas we can observe water infiltrations in the walls and the stained aspect suggests development of moulds and probably some species of bacteria. The fact that not even the accommodation surface is properly maintained is even more serious and judging by its aspect it seems not to have been periodically disinfected as the hygiene standards for swine shelters regulate. In some stalls (Fig. 4), we can sometimes observe large surfaces in which the floor appears to have a whitish or greyish color, which suggests the development of moulds on surfaces that come in direct contact with animals. Moreover, administering the green fodder directly on the floor favours their contamination through direct contact with moulds. Moulds on the floor can extend through continuity to the concrete feeding areas and thus come in contact with the combined fodder from pigs' alimentation. According to Koshinsky and Khachatourians (1992) the presence of fungi that produce toxins in animal food or the accommodating areas does not automatically imply toxicity. When more fungi are present, each of them can produce different mycotoxins and this combination can cause more adverse effects in comparison to only one mycotoxin because of the additive or synergic interaction. In this manner, coming in contact with various mycotoxins from moulds, even if they do not produce zearalenone, sensitize the animal, rendering it more vulnerable to estrogenic mycotoxins' action (e.g. zearalenone) when consuming Fusarium infested fodder.

Assessing the sows for reproduction highlighted that they are relatively non-uniform hybrids, which the owner bought on market. They are well taken care of, so that from this point of view, one could not state that the animals have problems. They are active, have a good appetite, thus judging from a clinical point of view, they seem healthy, but are a little underweight. The problems, which the owner confronted, are mainly linked to the reproductive activity. Sows presented infertility, comportamental changes (prolonged oestrus). The surprisingly small number of piglets farrowed in the majority of sows stood out easily. It is in truth suspicious to obtain 2-3 piglets in the majority of the cases, mostly small, from hybrid productive sows, well taken care of and with a adequate weight to the age category. Moreover, in some of them, beside the fact that they farrowed a small number of piglets, they were uneven and in some cases, they died in the first week of life. A possible explanation for these problems could be the presence of mycoestrogens (zearalenone) in the food of these animals, swines being the most sensitive species to this mycotoxin. Thus, the phenotypical aspect of sows, their decrease 
in weight, infecundity and small number of piglets, the histological changes allowed us to reach the conclusion that these moulds contained micoestrogens. When this mycotoxin is present in the alimentation of reproduction sows, the most common pathogenic effects are anestrus, abortion, increased embryonic and fetal death (Andretta et al., 2008). The histological changes present in ovaries justify to a large extent the infecundity and low prolificity of the sows. The ovarian activity was influenced by estrogens, possibly both from food and ovarian (secreted by the cysts). Since the oocyte degenerated under micoestrogenic influence, the reproductive activity consequently decreased. Various studies examined the possible impact of zearalenone during gestation period. Pregnant gilts and sows, which received an experimental dose of 2.8-3.0 ppm zearalenone in food, especially during early gestation, had smaller piglets (Christensen et al., 1972). Estrogenic stimulation determined by zearalenone interferes with the endometrium's secretory response to progesterone $\left(\mathrm{P}_{4}\right)$ during embryionic implantation in days 11-12 of gestation. If the zearalenone dose in sows exceeds $25 \mathrm{ppm}$, its action becomes drastic, leading to farrowing dead litters or neonatal mortality (Christensen et al., 1972).

The most critical period, in which zearalenone determines the highest rate of embryonic death, is between 7-10 days of gestation. Experimental administration of zearalenone in doses of $1 \mathrm{ppm}$ in sows during this period of gestation resulted in increased embryonic degeneration starting with day 9 , and the remaining blastocysts degenerated until day 13 (Long et al., 1992). The authors assume that this is due to the fact that zearalenone has an impact on secretory mechanism of the endometrium, modifying the intrauterine environment during early gestation. Interesting results were reported by Long et al. (1982), who concluded that levels of zearalenone higher than 64 ppm determine the death of all litters, while levels below 60 ppm lead to obtaining a small number of piglets, which are less vigorous. The authors reasoned this dose-related effect, claiming that the number of sows which farrow a small number of litter (1-3 piglets) increases with the higher the dose of zearalenone $(0,7,38$ and $64 \mathrm{ppm})$.

Few aspects are known about the effect of mycotoxins in piglets when feeding lactating sows with zearalenone contaminated fodder.
Piglet mortality during the first two weeks after farrowing was shown to be higher in sows that received fodder with $4.8 \mathrm{ppm}$ zearalenone during pregnancy and lactation (Kanora and Maes, 2009).

Other authors observed that administration of zearalenone in 5-10 ppm dose in sows after weaning leads to anestrus (Meyer et al., 2000), affirming that there is a linear relation between the dose of zearalenone in ppm and the length of anestrus in days. Same authors claim that some sows experience prolonged oestrus after farrowing.

According to the owner affirmations, we detected that some sows have a prolonged cycle and do not get pregnant, which resembles the claims made by the previously mentioned authors. Not finding a resolution to this problem, the owner receded from keeping these sows for reproduction, considering that the only economical solution to this problem was through sacrification.

\section{CONCLUSIONS}

Sows were confronting a persistent hyperestrogenism situation judging by the comportamental changes, phenotypical aspect, infertility and histological changes in ovaries and oviduct, possibly because of both the moulds from fodder and the ones secreted by the ovarian cysts, with continuous mobilisation of follicles from the follicular pool.

In the farm taken into study, there were completely inadequate conditions of storing the cereals and pluvial waters infiltrations in the spaces dedicated for preparation of combined fodder, aspects that favour the development of moulds.

Because of the contamined fodder and the inappropriate accommodation area, the reproductive efficiency of sows was considerably diminished.

\section{REFERENCES}

1. Adams NR (1995). Detection of the effects of phytoestrogens on sheep and cattle. Journal of Animal Science 73:1509-1515.

2. Andretta I, Lovatto PA, Hauschild L, Dilkin P, Garcia GG, Lanferdini E, Cavazini NC, Mallmann CA (2008). Feeding of pre-pubertal gilts with diets containing zearalenone. Arquivo Brasileiro de Medicina Veterinaria e Zootecnia 60:1227-1233.

3. Christensen CM, Mirocha CJ, Nelson GH, Quast JF (1972). Effect of young swine of consumption of rations containing 
corn invaded by Fusarium roseum. Applied Microbiology 23:202.

4. Desjardins AE (2006). Fusarium mycotoxins: Chemistry,Genetics and Biology. APS Press, St. Paul, Minnesota, p.260.

5. Diekman MA, Green ML (1992). Mycotoxins and reproduction in domestic livestock. Journal of animal science 70(5):1615-1627.

6. Hussein HS, Brasel JM (2001). Toxicity, metabolism, and impact of mycotoxins on humans and animals. Toxicology 167:101-134.

7. Kanora A, Maes D (2009). The role of mycotoxins in pig reproduction: a review. Veterinarni Medicina 54(12):565576.

8. Koshinsky HA, Khachatourians GG (1992). Trichothecene synergism, additivity and antagonism: the significance of the maximally quiescent ratio. Natural Toxins 1:38-47.

9. Long GG, Diekman M, Tuite JF, Shannon GM, Vesonder RF (1982), Effect of Fusarium roseum corn culture containing zearalenone on early pregnancy in swine, American Journal of Veterinary Research, 43, p. 1599-1603.
10. Long GG, Turek J, Diekman MA, Scheidt AB (1992). Effect of zearalenone on days 7 to 10 post-mating on blastocyst development and endometrial morphology in sows. Veterinary Pathology 29:60-67.

11. Meyer K, Usleber E, Martlbauer E, Bauer L (2000). Occurence of zearalenone, alpha and beta - zearalenone in bile of breeding sows in relation to reproductive performances. Berliner und Munchener Tierartzliche Wochenschrift 113:374-379.

12. Mirocha CJ, Christensen CM (1974). Oestrogenic mycotoxins synthesized by Fusarium. In: Purchase, I F. H. (ed.) Mycotoxins, Elsevier, p. 142-144, Amsterdam.

13. Shier WT, Shier AC, Xie W, Mirocha CJ (2001). Structureactivity relationships for human estrogenic activity in zearalenone mycotoxins. Toxicon 39:1435-1438.

14. Zinedine A, Soriano JM, Molto JC, Manes J (2007). Review on the toxicity, occurence, metabolism, detoxification, regulations and intake of zearalenone: an oestrogenic mycotoxin. Food Chem Toxicol. 45(1):1-18. 